\title{
Le chaînon manquant : la compréhension de la relation personne - groupe social - environnement
}

Diane Pruneau, Omer Chouinar et Hélène Gravel

\author{
(2) OpenEdition \\ Journals \\ Édition électronique \\ URL : https://journals.openedition.org/ere/7345 \\ DOI : $10.4000 /$ ere.7345 \\ ISSN : 2561-2271 \\ Éditeur \\ Centr'ERE
}

Référence électronique

Diane Pruneau, Omer Chouinar et Hélène Gravel, « Le chaînon manquant : la compréhension de la relation personne - groupe social - environnement », Éducation relative à l'environnement [En ligne], Volume 1 | 1999, mis en ligne le 15 septembre 1999, consulté le 28 mai 2021. URL : http:// journals.openedition.org/ere/7345; DOI : https://doi.org/10.4000/ere.7345 


\title{
Le chaînon manquant : la compréhension de la relation personne - groupe social - environnement
}

\author{
Diane Pruneau, Omer Chouinar et Hélène Gravel
}

1 Comment l'éducation peut-elle stimuler la construction de rapports engagés et significatifs entre les personnes, les groupes sociaux et l'environnement? Malgré l'évolution de l'éducation relative à l'environnement (ERE), la réponse à cette question demeure incomplète et vague. Diverses interventions pédagogiques ont été proposées : améliorer les connaissances des citoyens au sujet du fonctionnement des écosystèmes et des problèmes environnementaux, les impliquer directement dans l'action environnementale (Stapp et coll., 1988), favoriser l'acquisition de valeurs environnementales (Unesco - PNUE, 1985), encourager la prise de conscience sensorielle et affective des bienfaits du milieu naturel (Van Matre, 1990), habiliter les gens à entrevoir et choisir leur futur (Hicks, 1996), promouvoir leurs habiletés critiques pour évaluer les comportements et choix sociétaux (Sauvé, 1997), etc. La proposition de ces différentes interventions pédagogiques favorise la poursuite des objectifs généraux de l'ERE tels que formulés à la Conférence de Tbilissi en 1977: prise de conscience, connaissances, état d'esprit (attitudes), compétences et participation. Malheureusement, ces diverses interventions pédagogiques ont été peu évaluées quant à leur efficacité et à leur impact.

2 Par ailleurs, l'ERE évolue et se transforme, inspirée par les progrès de la recherche dans ses deux composantes : l'éducation et l'environnement. Moins exclusivement orientée vers la résolution immédiate des problèmes environnementaux, l'éducation relative à l'environnement nous apparaît maintenant comme un processus à long terme, durant lequel les apprenants, reconnus et respectés dans leurs savoirs, valeurs et décisions, sont invités à analyser l'état de leur milieu et leur relation avec celui-ci, puis à effectuer 
des choix personnels qu'ils jugent appropriés pour la construction d'un environnement meilleur.

3 Cette tendance actuelle de l'ERE peut être illustrée par les exemples suivants, présentés en lien avec les objectifs généraux de Tbilissi :

- Grâce à la théorie du biorégionalisme (Sale, 1991), la prise de conscience ne se limite plus à la saisie des problèmes environnementaux, mais également à la conscience réfléchie de la valeur et de l'importance des ressources, de la culture et des compétences locales. En effet, le biorégionalisme suppose la réhabitation de sa région telle que définie par ses frontières géographiques. Le biorégionalisme reconnaît, encourage et célèbre les liens locaux des personnes avec la terre, les plantes, les animaux, les cours d'eau, les familles, amis et voisins, les traditions indigènes et les systèmes de production et de commerce. Le mouvement biorégional vise à recréer une identité régionale fondée sur la conscience et le respect de l'intégrité de la communauté écologique locale.

- Influencé par les théories du socioconstructivisme et de l'apprentissage expérientiel en éducation (Pruneau et Lapointe, sous presse), l'objectif de l'acquisition de connaissances réfère maintenant à une connaissance du milieu construite grâce à un vécu sur le terrain et à un dialogue constant entre les savoirs traditionnels et scientifiques des acteurs sociaux.

- L'objectif du développement d'un état d'esprit s'enrichit quant à lui de la recherche d'attitudes telles que le sentiment de "pouvoir agir » (empowerment) issu des théories du développement local viable (Gagnon, 1994; Jean, 1997), la sollicitude (à l'origine associée à l'éducation morale) et l'identité écologique (Thomashow, 1995) ou communautaire (Hummon, 1992), provenant du domaine de la sociologie de l'environnement. Le sentiment de pouvoir agir est souvent la conséquence d'interventions régionales durant lesquelles les animateurs favorisent le renforcement et le développement des savoirs et savoir-faire locaux. La sollicitude est issue de la théorie éthique de Gilligan (1982) qui a découvert ce désir de prendre soin chez des sujets féminins et chez plusieurs sujets masculins. Or, cette valeur a été retrouvée chez plusieurs environnementalistes (Shogan, 1988). L'identité écologique fait référence à un lien d'attachement et à une identification aux éléments biophysiques de sa région alors que l'identité communautaire se définit comme l'interprétation du soi qui utilise la communauté comme centre d'attachement ou comme une image pour la caractérisation de soi. Ces deux types d'identité sont des composantes de l'attachement aux lieux qu'on retrouve chez certains individus impliqués dans des actions environnementales (Pruneau et Chouinard, 1997).

- Quant à l'objectif du développement de compétences, il peut aujourd'hui inclure des habiletés de leadership, d'entrepreneuriat et de prise en charge, habiletés mises de l'avant dans les domaines de l'alphabétisation en éducation des adultes (Wrigley, 1993), de la sociologie de l'environnement (Tessier et Vaillancourt, 1996) et du développement communautaire (Sragge, 1993 ; Favreau et Lévesque, 1996).

- Enfin, l'objectif de participation peut faire référence désormais à la surveillance des écosystèmes puisque des indicateurs de qualité environnementale ont maintenant été identifiés, même pour le milieu urbain (Lisowsky, 1998). Par ailleurs, des organismes internationaux ont créé des modes de participation communautaire de restauration environnementale tels que la méthode accélérée de recherche participative (Chambers, 1994) et l'analyse participative pour l'action communautaire (Peace Corps, 1996). Ces méthodes permettent à des chercheurs extérieurs à un milieu donné de recueillir de l'information au sujet des écosystèmes locaux en collaboration avec les acteurs de la région puis d'impliquer ces mêmes acteurs dans un processus de prise en charge de leur environnement. Les techniques ici utilisées pour le partage et l'appropriation de 
l'information régionale sont originales (par exemple, cartographie participative ${ }^{1}$, marches transversales ${ }^{2}$, sériation de situations et de solutions ${ }^{3}$, calendriers saisonniers ${ }^{4}$, étude des changements et des tendances ${ }^{5}$, etc.) et elles permettent la réalisation concrète de l'objectif général de participation. La participation devient multisectorielle et interdisciplinaire. Enfin, le partage global des informations et données recueillies au niveau local est d'ailleurs facilité par les récents développements en technologie éducative (Heimlich, 1998).

Comme on peut le constater dans la littérature et lors de conférences internationales telle la conférence de la NAAEE (North American Association for Environmental Education) en 1998, les objectifs de l'éducation relative à l'environnement se diversifient de même que les méthodes qui favorisent leur réalisation. Les spécialistes classifient et critiquent ces méthodes. Ce bouillonnement d'idées, qui est souhaitable puisque l'ERE participe à la réforme des modèles éducatifs (Sauvé, 1996), révèle chez les chercheurs un désir de découvrir des moyens d'améliorer les relations-personne groupes sociaux - environnement. Toutefois, il nous apparaît essentiel que la recherche en ERE s'intéresse à l'identification des effets spécifiques des diverses interventions pédagogiques proposées. Quelles sont la nature et la structure des expériences de vie favorisées par ces interventions? Quel est le sens attribué par les apprenants à l'environnement et aux expériences éducatives vécues? Quel est l'impact de ces interventions sur la représentation de l'environnement? Quelles sont les composantes de la relation à la communauté ou à l'environnement qui sont modifiées? Toutes ces questions pourraient donner lieu à des projets de recherche pertinents. De même, des méthodes d'investigation telles la phénoménologie, l'anthropologie, l'ethnométhodologie et la théorisation ancrée qui visent la compréhension profonde des phénomènes vécus nous apparaissent adaptées à ce type de questionnement, car elles valorisent la subjectivité et résultent fréquemment en la découverte d'éléments nouveaux.

5 La recherche sur les impacts des diverses méthodes pédagogiques permettra d'en évaluer la pertinence, d'associer à chacune une population apprenante optimale (en termes d'âge et de région géographique) et d'identifier leurs éléments de complémentarité. Les chercheurs risquent toutefois de rencontrer une limite importante en tentant d'interpréter les résultats de ces études. Ainsi, des chercheurs pourront observer, au cours d'une investigation particulière, que des activités impliquant l'exercice de la pensée critique augmentent l'habileté des apprenants à réfléchir au mode de vie des résidents de leur quartier ou que des activités d'exploration sensorielle rapprochent affectivement ces personnes du milieu naturel. Une habileté et une attitude semblent s'être développées, mais par rapport à quelle situation initiale? À l'heure actuelle, les chercheurs disposent de peu d'information sur la situation "avant l'apprentissage ", c'est-à-dire sur la relation que les personnes entretiennent quotidiennement avec leur communauté et leur environnement. Dans une société où les gens vivent rapidement, souvent sans porter attention au milieu biophysique, comment se porte la relation à l'environnement ? Les personnes sont-elles attachées à leur milieu de vie ? Existe-t-il une typologie des relations au milieu naturel, des relations au milieu construit? L'identité communautaire est-elle présente et estelle associée à des milieux particuliers? Les valeurs morales démontrées par des personnes ont-elles des répercussions sur leurs valeurs écologiques? Quels types d'éthique de l'environnement peuvent être rencontrés chez les environnementalistes? Quelles sont les idées des gens à propos de l'environnement? L'étude de ces questions 
chez différents groupes d'âge et dans diverses régions permettra aux éducateurs de l'environnement de tracer un portrait de différentes populations et de planifier des interventions pédagogiques adaptées à divers contextes relationnels.

6 Le questionnement présenté dans cet article est celui qui est partagé quotidiennement par notre équipe de recherche interdisciplinaire, composée d'une éducatrice, d'un sociologue, d'une spécialiste des nouvelles technologies et d'éthiciens. Ce questionnement donne naissance à un processus dynamique et non linéaire de va-etvient entre la recherche à propos des effets des interventions pédagogiques en ERE et l'étude fondamentale de la relation que les personnes établissent et entretiennent avec leur milieu biophysique et leur communauté. De façon plus spécifique, nous avons entrepris deux programmes de recherche. L'un se déroule dans la communautélaboratoire de Cap-Pelé. À cet endroit, nous expérimentons, auprès de groupes scolaires et communautaires, diverses interventions pédagogiques et nous mesurons leur impact sur plusieurs aspects de la relation à l'environnement: l'attachement aux lieux, l'identité communautaire, la motivation à l'action environnementale, etc. (Pruneau et coll., 1998; Pruneau et coll., sous presse). De même, une recherche portant sur la compréhension de la relation à l'environnement et de ses implications éthiques a été amorcée. Cette recherche phénoménologique, menée auprès d'adultes, vise la description de leur expérience quotidienne de l'environnement et de leur processus de décision éthique lorsqu'ils sont confrontés à un problème environnemental.

$7 \quad$ Les acteurs interrogés dans le cadre de ces études ont exprimé le vécu de leur relation avec le milieu de vie. Toutefois, la nature, la structure et l'intensité de cette relation diffèrent. L'attachement aux composantes des paysages et les raisons et manifestations du lien varient également. Le désir d'implication dans l'action environnementale semble parfois facile à susciter et parfois plus ardu. Les derniers résultats obtenus nous incitent à croire en la capacité des personnes d'améliorer leur relation avec le milieu et leur réflexion au sujet de leur pratique environnementale. D'autres recherches, dans le milieu et avec des personnes, seront toutefois essentielles autant en ce qui concerne l'évaluation des méthodes pédagogiques que la compréhension de la relation à l'environnement, afin d'identifier et comprendre les attitudes et comportements de nos sociétés face à l'espace et aux regroupements humains et afin d'élaborer des théories au sujet des interventions pédagogiques en ERE.

\section{BIBLIOGRAPHIE}

Chambers, R. (1994). Participatory Rural Appraisal (PRA) : Challenges, potentials, and pa-radigm. World Development, 22(10), 17-23.

Favreau, L. et Lévesque, B. (1996). Développement économique communautaire : économie sociale et intervention. Sainte-Foy : Presses de l’Université du Québec.

Gagnon, C. (1994). La recomposition des territoires, développement local viable. Paris : L'Harmattan.

Gilligan, C. (1982). Une si grande différence. Paris : Flammarion. 
Heimlich, J. (1998). Instructional and information technology - Where does EE fit in ? Communication présentée à la Conférence annuelle de la NAAEE, Atlanta, septembre 1998.

Hicks, D. (1996). Envisioning the future : The challenge for environmental educators. Environmental Education Research, 2, 101-108.

Hummon, D.M. (1992). Community attachment. Dans I. Altman et S.M. Low (dir.), Place Attachment. New York : Plenum Press. p. 253-278.

Jean, B. (1997). Territoires d'avenir. Ste-Foy : Presses de l'Université du Québec.

Lisowski, M. (1998). Expedition to Plan-it Earth. Pairing learning and nature with innova-tion technology. Communication présentée à la Conférence annuelle de la NAAEE, Atlanta, septembre 1998.

Peace Corps. (1996). Training manual PACA : Participatory Analysis for Community Action. Washington, D.C. : Peace Corps Information and Collection and Exchange.

Pruneau, D. et Lapointe, C. (sous presse). Un, deux, trois ? Nous irons aux bois... L'apprentissage expérientiel et ses applications en éducation relative à l'environnement. Dans R. Landry (dir.), Collectif sur la pédagogie actualisante, Moncton, NB : Presses de l'Université de Moncton.

Pruneau, D. et Chouinard, O. (1997). Un modèle d'intervention pédagogique qui favorise la relation personne - groupe social - environnement. Dans M. Hrimech et F. Jutras (dir.), Défis et enjeux de l'éducation dans une perspective planétaire. Sherbrooke, Québec : Éditions du CRP. p. 107-123.

Pruneau, D., Chouinard, O. et Musafiri, J.-P. (sous presse). Community groups and envi-ronmental action - Motivational factors. Dans NAAEE (dir.), Proceedings of the 27th Annual Conference of the North American Association for Environmental Education. Troy, OH : NAAEE.

Pruneau, D., Chouinard, O. et Arsenault, C. (1998). The Cap-Pelé Model. Alternatives Journal, 24 (3), 28-31.

Sale, K. (1991). Dwellers in the land. Philadelphie : New Society Publishers.

Sauvé, L. (1997). L'approche critique en éducation relative à l'environnement : origines théoriques et applications à la formation des enseignants. Revue des sciences de l'éducation, 23 (1), 169-188.

Sauvé, L. (1996). Alternatives cognitives pour le développement d'une responsabilité sociale : les propositions de l'éducation relative à l'environnement. Dans M. Rafie (dir.), Les sciences humaines : état des lieux. Ste-Foy, Québec : Les Presses de l'Université Laval, p. 171-182.

Shogan, D. (1988). Care and moral motivation. Toronto : OISE Press.

Sragge, E. (1993). Community economic development. Dans search of empowerment. Montréal : Black Rose Books.

Stapp, W., Bull, J., Cromwell, M., Cwikiel, J.W., Dichiro, G., Guarino, J., Rathje, R., Wals, A. et Youngquist, M. (1988). Education in action. A community problem solving program for schools. Dexter, MI : Thomson-Shore.

Tessier, R. et Vaillancourt, J.G. (1996). La recherche sociale en environnement : nouveaux paradigmes. Montréal : Presses de l'Université de Montréal.

Thomashow, M. (1995). Ecological identity : Becoming a reflective environmentalist. Cambridge, MA : MIT Press.

Unesco-PNUE. (1985). A guide on environmental values education. Environmental Educa-tion Series, no. 13. Paris : Unesco. 
Van Matre, S. (1990). Earth education. A new beginning. Warrenville, IL : The Institute for Earth Education.

Wrigley, H.S. (1993). Innovative programs and promising practices in adult ESL literacy. Washington, DC : National Clearinghouse on Literacy Education.

\section{NOTES}

1. La cartographie participative consiste à faire dessiner, par les acteurs sociaux, une représentation graphique de leur région où ils indiquent à l'aide de symboles (roches, bouts de bois et autres objets du milieu) les manifestations d'un problème (exemple: composantes du problème de la qualité de l'eau potable).

2. Les marches transversales consistent à arrêter le long d'une route, à des endroits stratégiques, et à marcher à angle droit par rapport à la route, pour identifier les composantes d'un problème et interroger les gens au sujet de ce problème.

3. Ici, les animateurs demandent aux participants de construire un tableau sur un sujet donné (exemple : les situations de bien-être dans la communauté) et d'accorder un nombre plus ou moins grand d'objets (haricots, bonbons, etc.) aux éléments qu'ils jugent les plus importants pour eux.

4. Les calendriers saisonniers sont des représentations graphiques des changements biophysiques et sociaux en fonction des saisons dans un milieu donné.

5. L'étude des changements et des tendances consiste à réunir des représentants des différents groupes sociaux de la communauté et à retracer avec eux le développement passé d'un problème environnemental et ses répercussions estimées pour le futur.

\section{AUTEURS}

\section{DIANE PRUNEAU}

Diane Pruneau est professeure à la Faculté des sciences de l'éducation de l'Université de Moncton (Nouveau-Brunswick, Canada). Elle enseigne la didactique des sciences et l'éducation relative à l'environnement. Ses principaux projets de recherche sont concentrés dans la région de Cap-Pelé qui sert de communauté-laboratoire pour l'étude de la relation à l'environnement et la mise en œuvre d'interventions pédagogiques menant à l'action environnementale.

\section{OMER CHOUINAR}

Omer Chouinard est professeur à l'Université de Moncton. Il a été agent de développement et de recherche auprès de coopératives et de syndicats dans les communautés côtières des Provinces maritimes. De même, à titre d'expert-conseil, il a été impliqué dans le développement d'outils de participation communautaire en vue de l'autonomisation des communautés de pêches au Nouveau-Brunswick. 


\section{HÉLÈNE GRAVEL}

Hélène Gravel est professeure au programme de la maîtrise en orientation à l'Université de Moncton. Elle fait de la recherche sur l'intégration des savoirs en éducation, le counseling vocationnel, la recherche phénoménologique et le vécu relationnel. 